\title{
Modelo de gestión integral para mejorar estándares educativos en escuelas de Chachapoyas
}

\section{Integral management model to improve the quality standards of educational in schools of Chachapoyas}

\author{
José Alex López Castro ${ }^{1}$, Lenin Del Castillo Chasquibol ${ }^{2}$
}

\section{RESUMEN}

La presente investigación tuvo como objetivos identificar los estándares educativos de las escuelas de Chachapoyas, Perú, identificar y analizar las teorías para dar sustento científico al modelo; diseñar un modelo de gestión integral para mejorar los estándares educativos de las escuelas de Chachapoyas; validar el modelo para lograr mejorar los estándares educativos de las escuelas de Chachapoyas, utilizando la técnica Delphy. Como materiales, se utilizó la tecnología, infraestructura, material impresos; se diseñó una encuesta administrada a los estudiantes, docentes y padres de familia. La metodología fue descriptiva y transversal, cuya población estuvo conformada por 29 docentes, 285 estudiantes y 207 padres de familia; con una muestra aleatoria estratificada de29 docentes, 72 estudiantes 66 padres de familia. Cuyos resultados, se registra: Uso de la información, en estudiantes: pocas veces $31,9 \%$; organización institucional 26,4\%; trabajo conjunto con las familias 18,1\%; soporte al desempeño docente $6,9 \%$. Padres de familia; organización institucional pocas veces, 59,1\%; uso de la información $54,5 \%$; soporte a desempeño docente 50,0\%; trabajo conjunto con los padres de familia $28,8 \%$. Docentes, pocas veces $65,5 \%$; soporte al desempeño docente $44,8 \%$, trabajo conjunto con las familias; $20,7 \%$, uso de la información con el 17,2\%.

Palabras claves: Modelo de gestión, estándares educativos

\begin{abstract}
This research has the following objectives identify the educational standards of the chachapoyas' schools, in Peru, identify and analyze theories to give scientific support to the model; design a comprehensive management model to improve the educational standards of the chachapoyas' schools; validate the model, in order to improve the educational standards of the chachapoyas' schools, using the Delphy technique. A complete logistics team was used, as a material; a suvery was designed for students, teachers and parents. The methodology was descriptive and transversal, the population consistend in: 29 teachers, 285 students and 207 parents; with a stratified random sample of 29 teachers, 72 students, 66 parents. The results are: students using information, rarely 31,9\%, institutional organization 26,4\%, join work with families 18,1\%, support for teacher performance $6,9 \%$. Regarding parentes: institutional organization, rarely 59,1\%, use of information 54,5\%, support for teacher performance $50,0 \%$, join work with parents $28,8 \%$. Regarding teachers: institutional organization, rarely $65,5 \%$, support for teacher performance $44,8 \%$, join work with families $20,7 \%$, use of information $17,2 \%$.
\end{abstract}

Keywords: Management model, educational standards

\footnotetext{
Dr. En Gestión Pública y Gobernabilidad. Profesor de la Escuela de Postgrado de la Universidad Nacional Toribio Rodríguez de Mendoza de Amazonas. Correo electrónico: josealex_lopezc@hotmail.com. http://orcid.org/0000-0002-0744-008X).

${ }^{2}$ Bachiller en educación. Profesor en la Institución Educativa Seminario Jesús María, Amazonas, Perú. Correo electrónico:lenindcc@gmail.com.
} 


\section{INTRODUCCIÓN}

La presente investigación titulada modelo de gestión integral para mejorar los estándares educativos de las escuelas de Chachapoyas, tiene por objetivo general, determinar en qué medida el modelo de gestión integral, servirá para mejorar los estándares educativos de Chachapoyas. Los datos se obtuvieron de una muestra de 29 docentes, 72 estudiantes y 66 padres de familia, haciendo un total de 167 personas de las escuelas sujeto a investigación. Se fundamenta en trabajos de investigaciones de diferentes espacios y escenarios; por citar: Patiño, Margarita S. (2016) En su tesis doctoral "Aportes a la Gestión Integral de las Instituciones Educativas Oficiales de Bogotá, a partir de los Resultados de la Evaluación del Impacto del Proyecto Educativo Líderes del Siglo XXI".

Cuyo objetivo general, se centró en poner en práctica políticas de gestión institucional innovadoras. Concluyendo; la gestión integral, implementada adecuadamente en las instituciones educativas, contribuyen significativamente a la mejora de la calidad educativa. De igual modo, García Poyato, Jihan R (2017) en su tesis titulada: "La gestión escolar como medio para lograr la calidad en instituciones públicas de educación primaria en Ensenada, Baja California" México; cuyo objetivo general, especifica, demostrar la importancia de la gestión escolar en instituciones públicas. Concluye; qué, la calidad educativa se gesta por una adecuada gestión escolar como política educativa, liderada por el personal directivo, administrativo, docente y padres de familia. Yábar, Imelda (2015) en su tesis de maestría: "La gestión educativa y su relación con la práctica docente en la Institución Educativa Privada Santa Isabel de Hungría de la Ciudad de Lima Cercado" Perú. Concluye; qué, la gestión escolar planificada, implementada y ejecutada con visión de innovación, responde a cumplir metas y necesidades sociales, por impartir una educación centrada en la calidad, a partir del personal que lideran dichos procesos educativos.

En esa perspectiva, lograr mejor los estándares educativos en las escuelas de Chachapoyas es una necesidad prioritaria; porque, permitirá tener servicios educativos de calidad, y se considera una condición necesaria para lograrlo.

Según, la evaluación PISA (2018), de 70 países evaluados, Perú se ubica en el 64 lugar, reflejándose en crisis educativa de impacto estructural. De acuerdo a Vexler Talledo, I. (2004), en su "Informe sobre la Educación Peruana, Situación y Perspectivas". Enfatiza diciendo que, "la crisis del sistema educativo, se centra por ausencia de un planteamiento estratégico en el sistema educativo peruano; cuyos logros de aprendizaje están por debajo de lo esperado y empeoran en las zonas pobres, rurales y con población con lenguas originarias". De igual modo; el Ministerio de Educación (2010), en la "Encuesta Diagnóstica"; enfatiza diciendo que, el número de estudiantes que se encuentran en riesgo de abandonar el sistema educativo es de 1" 058,457 estudiantes. A sí como, el 686,833 estudiantes se encuentran con atraso escolar de uno y dos años. se debe tener en cuenta que aproximadamente 259, 992 estudiantes desaprueban al menos un área, de los cuales repiten el grado 111, 632 estudiantes. La región Amazonas, no es ajena al bajo nivel educativo, según, SICRECE (2019) "UMC" de la Oficina de Medición de la Calidad de los Aprendizajes, los resultados de la ECE son: Lectura: El porcentaje de estudiantes con resultados satisfactorios fue del $43.1 \%$, el $49.4 \%$ estaban en proceso, y el $7.6 \%$, están en inicio o presentan deficiencias. Matemática: Se encontró que en el nivel satisfactorio estaba el $32.0 \%$ de los escolares, el $37.7 \%$ están en proceso y el $30.3 \%$ en inicio.

En esa lógica, poner en acción el modelo de gestión integral para mejorar los estándares educativos de las escuelas de Chachapoyas, fue prioritario. Las escuelas que brindan una educación eficiente a la sociedad, es porque sus estándares educativos son de calidad.

\section{MATERIALY MÉTODO}

La población universal, estuvo conformada por 521 personas entre estudiantes, docentes y padres de familia de las escuelas de Chachapoyas. Para determinar la muestra se utilizó el muestreo probabilístico estratificado con afijación proporcional, utilizando la siguiente fórmula:

$$
n_{o}=\frac{Z_{P}^{2} P Q N}{e^{2}(N-1)+Z^{2} P Q}
$$

\section{Donde:}

Z: Coeficiente de confianza asociado a la distribución $\mathrm{N}$ o r m a $1, \quad$ i g u a 1 a 1,96 para un nivel de confianza del 95\% (valor tabular de la distribución Normal).

$\boldsymbol{\alpha}$ : Nivel de significación igual a 0,05 (5\%)

P: Proporción de éxito igual a 0.5 asumiendo el criterio de varianza máxima

Q: Proporción de fracaso, igual a 1-P $=0.5$

$\mathbf{N}$ : Total de personas que conforman la Población

e: Error de muestreo, igual a 0,10 (asumida por el autor)

Para la población de estudiantes se reemplazó en la fórmula 1 obteniéndose el tamaño de muestra:

$$
\begin{aligned}
& n=\frac{(1,96)(0,5)(0,5)(285)}{(0,10)^{2}(285-1)+(1,96)^{2}(0,5)(0,5)} \\
& n=72 \text { Estudiantes. }
\end{aligned}
$$

De la misma forma para la población de padres de 
familia se reemplazó en la fórmula 1, obteniéndose el tamaño de muestra:

$$
\begin{aligned}
n_{\mathrm{bi}} & =\frac{(1,96)(0,5)(0,5)(207)}{(0,10)^{2}(207-1)+(1,96)^{2}(0,5)(0,5)} \\
n_{\mathrm{i}} & =\mathbf{6 6} \text { Padres de familia }
\end{aligned}
$$

Con respecto a los docentes, considerando que son una población pequeña se optó por trabajar como muestra a toda la población, es decir que la muestra fue considerada igual a la población.

\section{$\mathrm{n}=\mathbf{2 9}$ docentes}

Las muestras obtenidas fueron repartidas de forma proporcional a su tamaño poblacional, mediante la siguiente fórmula:

$n_{i}=n\left(\frac{N_{i}}{N}\right)$

\section{Diseño de la investigación}

El estudio fue de tipo descriptiva - propositiva porque se recogió información para los fines específicos de la investigación, utilizándose el enfoque cuantitativo de tipo propositivo.

El diseño utilizado fue:
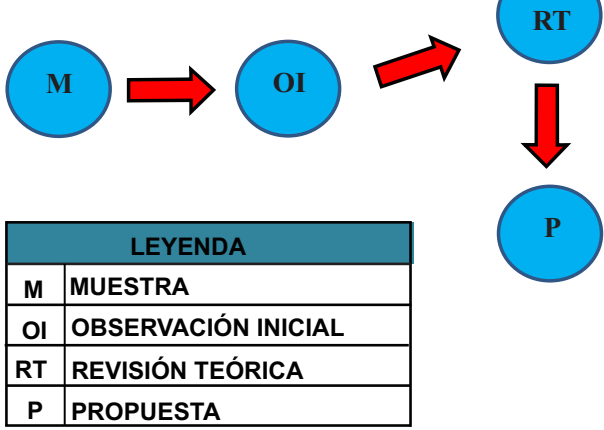

Los procedimientos realizados para alcanzar los objetivos propuestos, considerados:

Se administró una encuesta dirigida a los docentes, estudiantes y padres de familia, basada en preguntas específicas por dimensiones: soporte al desempeño docente, trabajo conjunto con las familias, organización institucional, Uso de la información; para Identificar los estándares educativos de las escuelas en estudio. Se realizó una revisión y análisis bibliográfico para dar sustento teórico al modelo de gestión integral. Posteriormente, se diseñó el modelo de gestión integral, para lograr mejorar los estándares educativos. Finalmente; se validó el modelo de gestión integral mediante juicio de expertos (tres doctores con mención en Gestión Pública) para garantizar la mejora de los estándares educativos en las escuelas en estudio, utilizando técnica Delphy.

Se consideró el siguiente planteamiento de hipótesis:
El modelo de gestión integral, servirá para mejorar los estándares educativos de las escuelas de Chachapoyas.

\section{RESULTADOS}

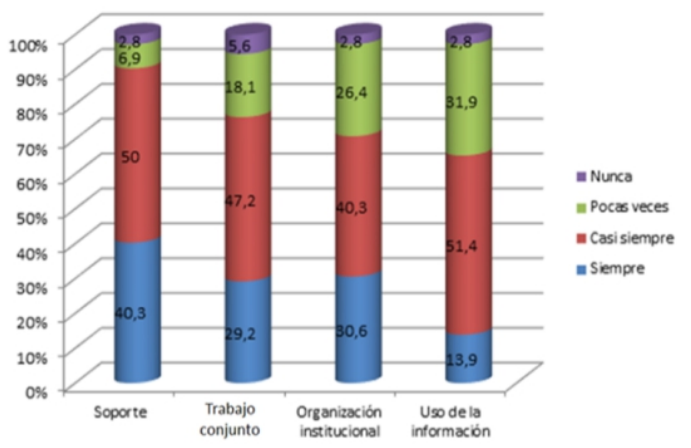

Figura 1. Distribuciones porcentuales de la percepción de los estudiantes acerca de los estándares de calidad.

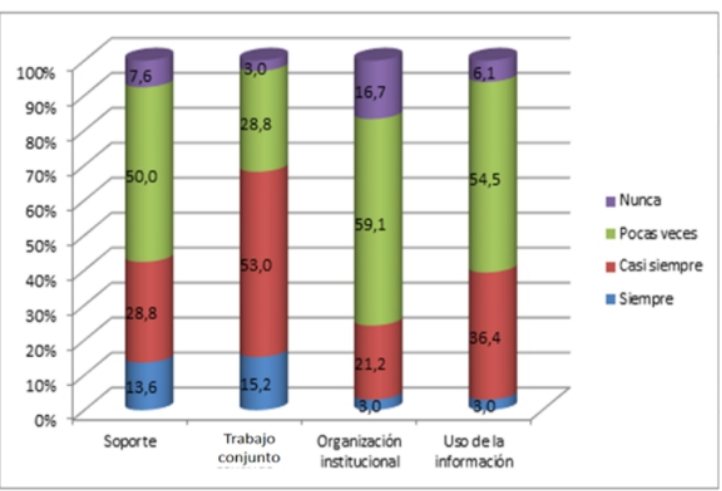

Figura 2. Distribuciones porcentuales de la percepción de los padres de familia, acerca de los estándares de calidad

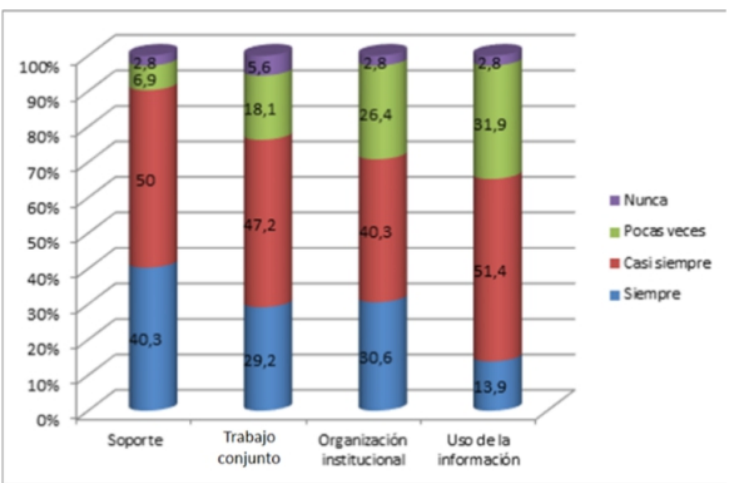

Figura 3: Distribuciones porcentuales de la percepción de los docentes, acerca de los estándares de calidad.

\section{DISCUSIÓN}

En la presente investigación "Modelo de gestión integral para mejorar los estándares educativos de las escuelas de Chachapoyas"; para comprobar la importancia del modelo en mención, se administró como instrumento de recolección de datos una encuesta validada por SINEACE-MINEDU (2014) a 
las escuelas en estudio a una población muestral de 176 personas; de las cuales: 29 docentes, 72 estudiantes y 66 padres de familia.

Procesada dicha información, los resultados nos llevan a confirmar la hipótesis de estudio planteada; el modelo de gestión integral, servirá para mejorar los estándares educativos de las escuelas de Chachapoyas. Con relación a la primera (figura 1) Distribuciones porcentuales de la percepción de los estudiantes acerca de los estándares de calidad. "Soporte al desempeño docente"; en la categoría siempre, se observa el $40,3 \%$, en casi siempre con el $50,0 \%$, pocas veces con el $6,9 \%$ y nunca con el $2,8 \%$. "Trabajo conjunto con las familias"; se observa en siempre, el $29,2 \%$, casi siempre, $47,2 \%$, pocas veces, $18,1 \%$ y nunca, 5,6\%. "Organización institucional"; siempre, con el 30,6\%, casi siempre, con el 40,3\%, pocas veces, con el $26,4 \%$ y nunca con el $2,8 \%$. "Uso de la información"; se observa en siempre, 13,9\%, casi siempre $51,4 \%$, pocas veces, $31,9 \%$ y nunca con el $2,8 \%$.

Dichos resultados, guardan relación con la tesis "Evaluación de programas de Educación: estándares de calidad", concluye: La necesidad de establecerla como responsabilidad prioritaria los estándares educativos de calidad; liderado por los entes educativos; estos, deberán responder a esta exigencia; y, con mayor razón el docente (Fontal, O. \& García, S. 2019). Así mismo, el Informe de PISA (2019) en el "Ranking de (Programme for International Student Assessment)," donde Perú, ocupó el último lugar en dicha evaluación. Es justamente, por la ausencia de los estándares de calidad en las escuelas de estudio y a nivel general.

(figura 2) Distribuciones porcentuales de la percepción de los padres de familia, acerca de los estándares de calidad. "Soporte al desempeño docente"; siempre, $13,6 \%$, casi siempre, $28,8 \%$, pocas veces, $50,0 \%$ y nunca, 7,6\%. "Trabajo conjunto con las familias"; siempre, $15,2 \%$, casi siempre, $53,0 \%$, pocas veces, $28,8 \%$ y nunca, $3,0 \%$. "Organización institucional"; siempre, 3.0\%, casi siempre, $21.2 \%$, pocas veces, $59.1 \%$ y nunca, $16.7 \%$. "Uso de la información"; siempre, 3,0\%, casi siempre, $36,4 \%$, pocas veces, $54,5 \%$ y nunca, $6,1 \%$. Estos resultados, guardan relación con la tesis "Participación de los padres de familia y su influencia en la gestión institucional en la Ugel de Canchis, Cusco". Concluye:

El nivel de participación de los padres de familia es deficiente en la gestión institucional de las entidades educativas de la Ugel de Canchis; esto se debe, la falta de organización de los padres de familia, y al estilo autoritario de las directoras/es del nivel de educación inicial, (Ramos Durán, Inés G, 2016).

Según, el MINEDU - ECE (2019): Del 94\% de estudiantes del $2^{\circ}$ grado evaluados, en Lectura: El
$49.8 \%$ lograron resultados satisfactorios, el $43.8 \%$ están en proceso, el $6.5 \%$ están en inicio. En Matemática sólo el $26.6 \%$ obtuvieron resultados satisfactorios, el $42.3 \%$ en proceso, el $31.0 \%$ en inicio. De igual manera, el $4^{\circ}$ grado de Secundaria, en el área de Lectura, los resultados que se obtuvieron no eran favorables, pues sólo el $27.0 \%$ eran satisfactorios. Reflejándose, la crisis educativa.

En la dimensión "organización institucional" (figura 3), Distribuciones porcentuales de la percepción de los docentes, acerca de los estándares de calidad. se observa que, en "Soporte al desempeño docente"; siempre, $10,3 \%$, casi siempre, $17,2 \%$, pocas veces, $44,8 \%$ y nunca, $27,6 \%$. "Trabajo conjunto con las familias"; siempre, $24,1 \%$, casi siempre, $51,7 \%$, pocas veces, $20,7 \%$ y nunca, 3,4\%. "Organización institucional"; siempre con el 6,9\%, casi siempre con el $17,2 \%$, pocas veces, $65,5 \%$ y nunca, $10,3 \%$. "Uso de la información"; siempre, 24,1\%, casi siempre, $48,3 \%$, pocas veces, $17,2 \%$ y nunca, $10,3 \%$. De igual manera, dichos resultados, guardan relación con el MINEDU, (2010) "Encuesta Diagnóstica", enfatiza qué, 1'058, 457 estudiantes estaban en riesgo de abandonar del sistema educativo. A ello se suma el 686,833 se encuentran con atraso de uno y dos años. De igual manera, 259, 992 estudiantes son desaprobados con al menos un área y repiten el grado 111, 632 estudiantes. Estos resultados reflejan que nuestra educación está atravesando una crisis, Vexler Talledo, I. (2004) "Informe sobre la Educación Peruana, Situación y Perspectivas". Enfatiza diciendo que:

"La crisis del sistema educativo, se centra por ausencia de un planteamiento estratégico en el sistema educativo peruano; cuyos logros de aprendizaje están por debajo de lo esperado y empeoran en las zonas pobres, rurales y con población con lenguas originarias". (MINEDU 2014, Marco del Buen Desempeño del Directivo, (p. 5)

\section{CONCLUSIÓNES}

1. Se propuso un modelo de gestión integral para lograr mejorar los estándares educativos en las escuelas de Chachapoyas.

2. Se logró diagnosticar los estándares educativos en las escuelas de Chachapoyas, siendo lo siguiente: Estudiantes, respondieron: Uso de la información, pocas veces, con el $31,9 \%$, seguido por la organización institucional con el 26,4\%, el trabajo conjunto con las familias y la comunidad el 18,1\% y soporte al desempeño docente con el 6,9\%. Padres de familia, respondieron: Organización institucional, pocas veces con el 59,1\%, seguido del uso de la información con 54,5\%; luego, soporte a desempeño docente con el 50,0\%; finalmente, el trabajo conjunto con los padres de familia y la comunidad, con el 28,8\%. Docentes, respondieron: Organización institucional, pocas 
veces con el $65,5 \%$, soporte al desempeño docente, con el 44,8\%; luego, el trabajo conjunto con las familias y la comunidad con el $20,7 \%$ y finalmente, el uso de la información con el 17,2\%. Hecho que se evidencia el estado crítico de los estándares educativos en las escuelas de Chachapoyas. en estudio.

3. Se identificó y analizó las siguientes teorías: El constructivismo, pensamiento crítico, el aprendizaje basado en problemas, el aprendizaje por descubrimiento, entre otras. Cuyos principios pedagógicos y didácticos, no son tomados en cuenta.

4. Se diseñó y validó un Modelo de Gestión Integral para lograr mejorar los estándares educativos en las escuelas de Chachapoyas, utilizando la técnica Delphi, encontrándose un modelo viable y bueno para su implementación.

\section{REFERENCIAS BIBLIOGRÁFICAS}

Almaguer, T. (2000). La calidad en la educación pública. México

Alves, J (2000). Liderazgo y clima organizacional. Revista Psicología del Deporte. Báxter, E. (2000). La Formación de Valores, una

Tarea Pedagógica. La Habana, Cuba: Pueblo y Educación.

Cantú, C. (2012). La Calidad Educativa en Argentina desde una Perspectiva Económica (Tesis de Maestria). Mendoza, Argentina.

Cuatrecasas, L. (2000). La gestión de la calidad total. Barcelona, España.

Cuenca, R. (2001). Coordinador del programa PREDUCA SOBRE EL DESEMPEÑO DOCENTE.

DREA, Oficina de Estadística. (2012). Datos estadísticos. Chachapoyas.

Gobierno Regional Amazonas. (2007). Plan Cyma. Chachapoyas.

Gobierno Regional Amazonas. (2009). Plan de Desarrollo Regional Concertado. Chachapoyas.

Gobierno Regional de Amazonas. (2007). Proyecto Educativo Regional PER. Chachapoyas.

Ministerio de Educación UMC. (2015). Resultados de la Evaluación Censal ECE de los estudiantes a nivel nacional. Lima.

Ministerio de Educación SINEACE (2014) ¿Qué y cómo evaluamos la gestión de la institución educativa)? Lima

Siabato Patiño, M. (2013). Aportes a la Gestión Integral de las Instituciones Educativas Oficiales de Bogotá, a partir de los Resultados de la Evaluación del Impacto del Proyecto Educativo Líderes del Siglo XXI (Tesis Doctoral). Colombia: Uniersidad Cooperativa de Colombia.

Vásquez, A. (2013). Interdependencia entre el Liderazgo Transformacional, Cultura Organizacional y Cambio Educativo: Una Reflexión. Revista Iberoamericana sobre Calidad, Eficacia y Cambio en Educación, 11(1), $73-91$.

Vexler Talledo, I. (2004). Informe sobre la Educación Peruana, Situación y Perspectivas. Lima, Perú.

1. Villafranca, D. (2002). Metodología de la Investigación. España 